\title{
Chercheurs d'or et contamination par le mercure des systèmes aquatiques continentaux de Guyane - Risques à l'égard des populations humaines
}

\section{Goldmining activities and mercury contamination of freshwater systems in French Guiana - Risks towards human populations}

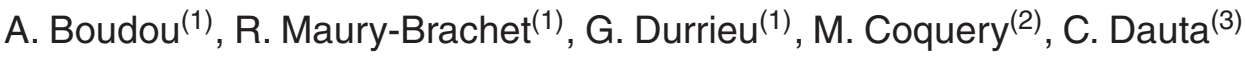 \\ (1) Laboratoire d'Ecophysiologie et Ecotoxicologie des Systèmes Aquatiques (LEESA), UMR CNRS 5805, \\ Université Bordeaux 1, Place Dr Peyneau, 33120 Arcachon, France \\ (2) Cemagref, groupement de Lyon, 69336 Lyon, France \\ (3) Centre d'Ecologie des Systèmes Aquatiques Continentaux (CESAC), UMR CNRS 5576, Université de \\ Toulouse III, 31 Toulouse, France
}

Résumé - La pollution par le mercure en Amazonie brésilienne et en Guyane française est principalement liée aux activités d'orpaillage, qui rejettent des quantités importantes de mercure élémentaire $\left(\mathrm{Hg}^{\circ}\right)$ lors des étapes d'amalgamation et amplifient les apports de $\mathrm{Hg}$ inorganique (Hgll) via l'érosion des sols et des sédiments, naturellement riches en métal. Dans le cadre du programme de recherche interdisciplinaire "Mercure en Guyane ", initié en 1997 par le CNRS/PEVS, une étude approfondie a été menée sur la contamination de la composante biologique des hydrosystèmes guyanais. Les résultats présentés dans cet article reposent sur une approche systémique, mise en œuvre au niveau de 3 sites à proximité du barrage hydroélectrique de Petit-Saut : 2 cours d'eau similaires au regard des caractéristiques hydrologiques mais différents par la présence ou l'absence d'activités d'orpaillage sur leur bassin-versant (criques Leblond et Courcibo, respectivement); une station à l'aval de la retenue, sur le fleuve Sinnamary. La bioamplification joue un rôle prépondérant et conduit à des concentrations de mercure dans le tissu musculaire des poissons carnivores/piscivores élevées, voire très élevées, par rapport à la norme de l'OMS (0,5 mg/kg, pds frais), alors que les niveaux de contamination de la colonne d'eau sont extrêmement faibles, de l'ordre du ng/L. Si les activités d'orpaillage contribuent à un accroissement très significatif des quantités de mercure au sein de la colonne d'eau, principalement sous forme particulaire, la comparaison entre les niveaux de contamination des poissons communs aux deux criques Leblond et Courcibo ne révèle pas de différences significatives. En fait, les concentrations de méthylmercure $(\mathrm{MeHg})$ dans la phase dissoute de la colonne d'eau sont comparables et peuvent expliquer cette surprenante similarité. Par contre, à l'aval de la retenue du barrage de Petit-Saut, les poissons sont nettement plus contaminés, et les concentrations en $\mathrm{MeHg}$ dans la colonne d'eau sont aussi beaucoup plus élevées. Ceci peut s'expliquer par les conditions anoxiques d'une large part de la colonne d'eau de la retenue qui conduisent à une forte production de $\mathrm{MeHg}$, via les bactéries sulfato-réductrices. Dans ces conditions, la consommation des poissons situés en fin de réseau trophique présente des risques pour les populations humaines, qui sont illustrés par les travaux 
récemment menés sur les communautés amérindiennes vivant dans la zone du Haut Maroni (villages Wayana), à la frontière entre la Guyane et le Surinam. Ceux-ci ont mis en évidence de forts niveaux d'imprégnation en mercure chez les enfants et les adultes, supérieurs aux limites recommandées par l'OMS.

Mots clés - orpaillage, mercure, méthylmercure, bioamplification, poissons, santé humaine

\begin{abstract}
Mercury pollution in the Amazon basin and in French Guiana is essentially linked to goldmining activities, with elemental mercury $\left(\mathrm{Hg}^{\circ}\right)$ release during the amalgamation step and increased inorganic mercury (Hgll) discharge through the erosion of soils and sediments, which are naturally rich in mercury. Within the multidisciplinary programme "Mercury in Guiana", set up in 1997 by the French CNRS/PEVS, a detailed study was set up in order to investigate mercury contamination of the biological component of freshwater systems. Here we present results from a systemic approach on three sites around the PetitSaut hydroelectric reservoir: two adjacent rivers, with and without goldmining activities on their watersheds (Leblond and Courcibo rivers, respectively) and one station located downstream of the dam on the Sinnamary river. Biomagnification plays a fundamental role and leads to very high $\mathrm{Hg}$ concentrations in the skeletal muscle of carnivorous/piscivorous fish, at the top of the aquatic foodwebs, although extremely low contamination levels were measured in the water column compartments ( $\mathrm{Hg}$ concentration close to the $\mathrm{ng} / \mathrm{L}$ or ppt level). Goldmining activities induce a significant and marked increase of $\mathrm{Hg}$ concentrations in the water, mainly under the particulate form. However, the comparison between fish species sampled simultaneously in Leblond and Courcibo rivers reveals no significant differences between $\mathrm{Hg}$ concentrations in fish muscle. In fact, methylmercury (MeHg) levels were similar in the dissolved phase of the water column for both rivers, which could explain the fish results. Downstream from the Petit-Saut reservoir, $\mathrm{Hg}$ concentrations in fish were significantly higher, in agreement with MeHg levels in the water column which were also markedly higher, owing to high methylation rates within the bottom anoxic water layers in the reservoir, via sulfur-reducing bacteria activity. Under these conditions, human populations that consume fish regularly and in large quantities, such as native Amerindian communities in the upper reaches of the Maroni river (Wayana villages), show high impregnation levels in children and adults, above the WHO safety limit.
\end{abstract}

Key words - goldmining, mercury, methylmercury, biomagnification, fish, human health

\section{INTRODUCTION}

Dans le bassin amazonien et en Guyane française, la pollution de l'environnement par le mercure est principalement attribuée aux activités d'orpaillage, ces dernières utilisant le $\mathrm{Hg}$ élémentaire $\left(\mathrm{Hg}^{\circ}\right)$ pour amalgamer les micro-particules de métal précieux, facilitant ainsi les rendements d'extraction (Malm, 1998; Villas Bôas, 1997). Malgré l'incertitude des données disponibles, plusieurs auteurs évaluent les émissions totales de mercure en Amazonie brésilienne, depuis la deuxième ruée vers l'or à la fin des années 80 , entre 1500 et 3500 tonnes, avec une moyenne proche de $200 \mathrm{t}$ par an (Lacerda \& Salomons, 1998). En Guyane française, la production officielle d'or entre 1857 et 1992 est estimée à $170 \mathrm{t}$, soit environ $230 \mathrm{t}$ de $\mathrm{Hg}^{\circ}$ perdues au cours des phases de lavage du minerai, de manipulation et de chauffage de l'amalgame sans recyclage des vapeurs métalliques (Picot 
et al., 1993). Toutefois, ces données correspondent à une sous-estimation importante de la production aurifère et des rejets de mercure élémentaire, compte-tenu de l'importance des activités clandestines dans ce domaine. Parallèlement à cette source anthropique directe de mercure, des apports peuvent provenir des processus d'érosion des sols, en relation avec les activités minières, la déforestation, les aménagements routiers et urbains ou les activités agricoles. En effet, les sols amazoniens sont naturellement riches en $\mathrm{Hg}$, les concentrations pouvant être plusieurs dizaines de fois supérieures à celles mesurées dans les sols des régions tempérées et boréales de l'hémisphère Nord. Ces sols amazoniens sont très anciens (jusqu'à plusieurs millions d'années) et ils ont accumulé, via des dépôts atmosphériques, des quantités importantes de métal, complexé avec les oxyhydroxydes d'aluminium et de fer (Grimaldi M. \& Grimaldi C., 2002; Roulet et al., 1998, 2000). Ainsi, l'érosion des particules au niveau des bassins-versants peut être à l'origine d'apports conséquents de mercure, principalement sous forme inorganique (Hgll), dans les hydrosystèmes, ces apports ayant une origine « naturelle». Enfin, des apports atmosphériques, dont une grande partie est d'origine anthropique, constituent aussi une source diffuse.

De nombreux travaux de recherche ont été menés au Brésil au cours des deux dernières décennies sur la contamination mercurielle dans le bassin de l'Amazone (rivières Tapajos et Madeira par exemple), dans le cadre de programmes nationaux et internationaux (Bidone et al., 1997; Boischio \& Henshel, 1996; Carmouze et al.,
2001; Dorea et al., 1998; Lacerda \& Salomons, 1998; Moreira, 1996; Reuther, 1994; Roulet et al., 1998, 2000, 2001; Veiga et al., 1999). En Guyane française, très peu de données scientifiques étaient disponibles (Richard et al., 1999, 2000 ; Roulet et Lucotte, 1995) avant la mise en place par le CNRS/PEVS du Programme de recherche pluridisciplinaire «Mercure en Guyane » (1997/2002). Ce programme $^{(1)}$ avait pour objectif principal l'analyse des composantes du cycle biogéochimique du mercure - sources, voies de transfert et devenir dans les compartiments abiotiques (air, sols, colonne d'eau/sédiments), contamination des réseaux trophiques (bioamplification) -, à partir de différentes zones d'étude (Fig. 1) : territoires amérindiens du Haut-Maroni; sites d'orpaillage de Dorlin et rivière Inini; barrage hydroélectrique de Petit-Saut, depuis le site d'orpaillage de Saint Elie en amont de la retenue, jusqu'au fleuve Sinnamary en aval. Des études ont également été menées à l'interface « environnement/santé », afin de quantifier les voies de transfert du mercure vers les populations humaines et d'appréhender les impacts, principalement neurologiques, sur les enfants (Frery et al., 1999, 2001; Cordier \& Garel, 1999).

Cet article présente les résultats relatifs à la contamination de la composante biologique des systèmes

(1) Ce programme de recherche a été financé par le CNRS/PEVS, le MATE et les fonds européens FEDER. II regroupe une douzaine de laboratoires, avec une Direction collégiale de 4 membres : L. Charlet (Université de Grenoble/CNRS), A. Boudou (Université Bordeaux 1/CNRS), M. Grimaldi (IRD) et D. Cossa (Ifremer). 


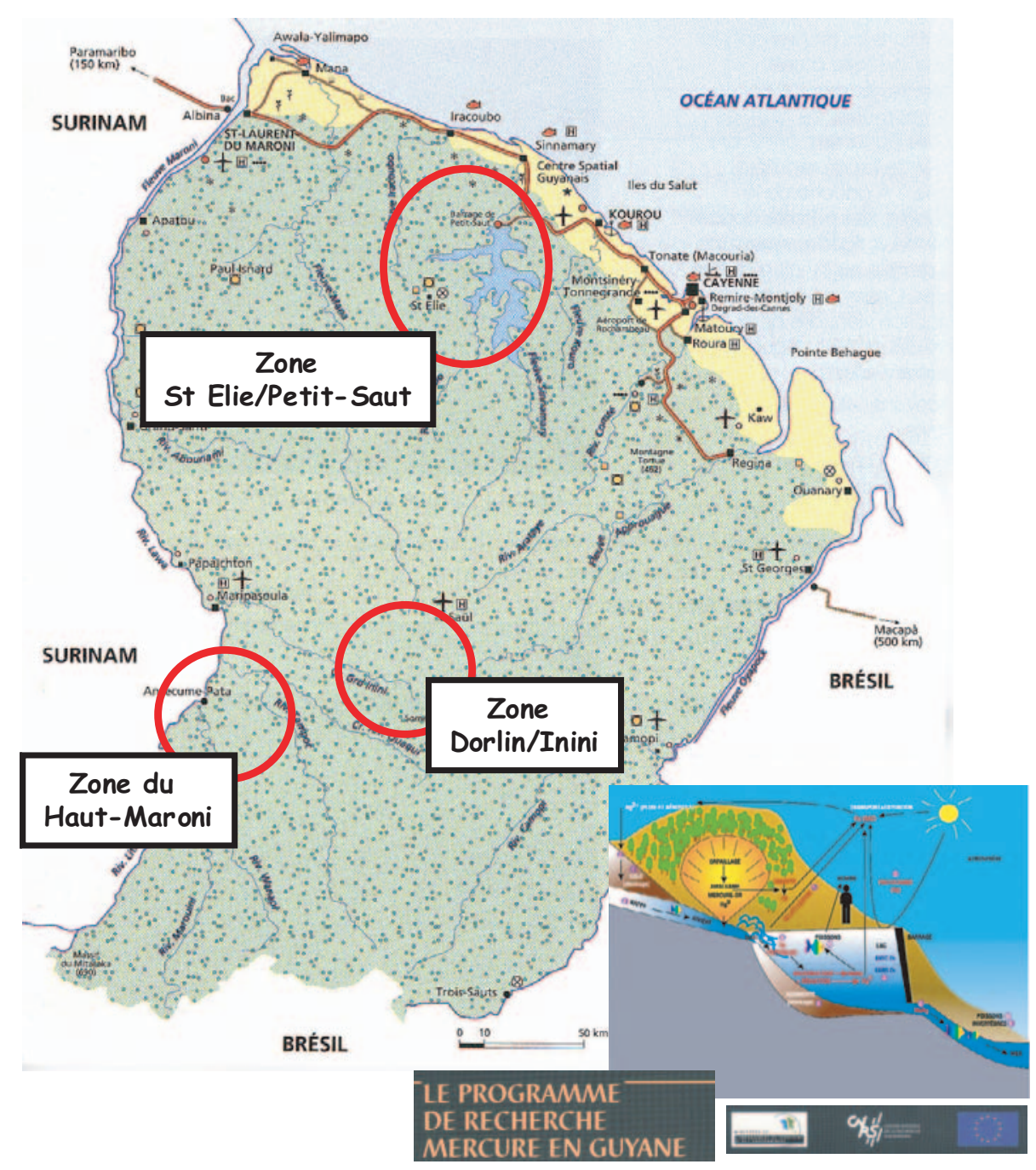

Fig. 1. Localisation des principaux sites d'étude dans le cadre du programme de recherche interdisciplinaire « Mercure en Guyane » (1997-2001).

Support cartographique : Atlas de Guyane (Zonzon et Prost, 1997).

Fig. 1. Study area in the "Mercury in French Guiana" research programme (1997-2001) (map: Atlas of Guyane, Zonzon and Prost, 1997). 
aquatiques continentaux de Guyane, en insistant plus particulièrement sur le concept-clef de la bioamplification du mercure le long des réseaux trophiques et sur le rôle prépondérant joué par la production et par la biodisponibilité de la forme mono-méthylée du métal (méthylmercure). Les résultats présentés proviennent des zones amont et aval de la retenue du barrage de Petit-Saut. Les relations entre la contamination des espèces aquatiques, plus particulièrement les espèces carnivores/piscivores, et les transferts trophiques vers les populations humaines seront également abordées, à partir des études réalisées sur les communautés amérindiennes Wayanas, au niveau des différents villages situés dans la zone du HautMaroni, à la frontière entre la Guyane et le Surinam.

\section{UN CONCEPT-CLEF : LA BIOAMPLIFICATION DU MERCURE LE LONG DES CHAÎNES TROPHIQUES AQUATIQUES}

La bioamplification du mercure à l'échelle des écosystèmes aquatiques se traduit par un accroissement généralement progressif des concentrations du métal accumulé dans les organismes, depuis la base des réseaux trophiques (producteurs primaires/végétaux) jusqu'aux consommateurs terminaux (espèces carnivores/piscivores) (Boudou \& Ribeyre, 1997 ; Mason et al., 1995 ; Morel et al., 1998; Wiener et al., 2002). Comme l'illustre le schéma de la figure 2 , ce processus de bioamplification dépend étroitement de la forme chimique du mercure et des mécanismes qui favorisent la production du monométhylmercure $\left(\mathrm{CH}_{3} \mathrm{HgX}\right.$ ou $\left.\mathrm{MeHg}\right)$. Le $\mathrm{MeHg}$ est un dérivé organique issu de la méthylation du $\mathrm{Hg}$ inorganique $(\mathrm{Hg}(\mathrm{II}))$, ce dernier résultant de l'oxydation du $\mathrm{Hg}$ élémentaire $\left(\mathrm{Hg}^{\circ}\right)$, utilisé lors de l'étape d'amalgamation de l'or.

La bioamplification repose sur les transferts cumulatifs du $\mathrm{MeHg}$ entre les proies et les prédateurs. $\mathrm{Le} \mathrm{MeHg}$ possède en effet une très forte capacité d'absorption au travers de la paroi du tractus digestif, proche de $100 \%$ chez les espèces aquatiques carnivores, et ensuite de séquestration dans les différents tissus biologiques, notamment le muscle squelettique chez les poissons (Boudou \& Ribeyre, 1997; Maury-Brachet et al., 2006 ; Wiener et al., 2002). Les conséquences écotoxicologiques de la bioamplification du mercure se traduisent par des accumulations extrêmement importantes dans les espèces situées en fin de chaînes alimentaires, alors que les niveaux de contamination dans l'eau sont très faibles, ne présentant aucuns risques directs pour l'homme, via la consommation de l'eau ou la baignade par exemple.

Les approches systémiques mises en œuvre dans le cadre de ce programme de recherche sur plusieurs cours d'eau guyanais, couplées aux études géochimiques sur le devenir du mercure dans les différents compartiments abiotiques (colonne d'eau, sédiments), ont permis une quantification détaillée de la bioamplification du mercure. La figure 3 illustre une partie des résultats obtenus sur la Crique Leblond, située en amont du barrage hydroélectrique de Petit-Saut et affectée par les activités d'orpaillage du site 


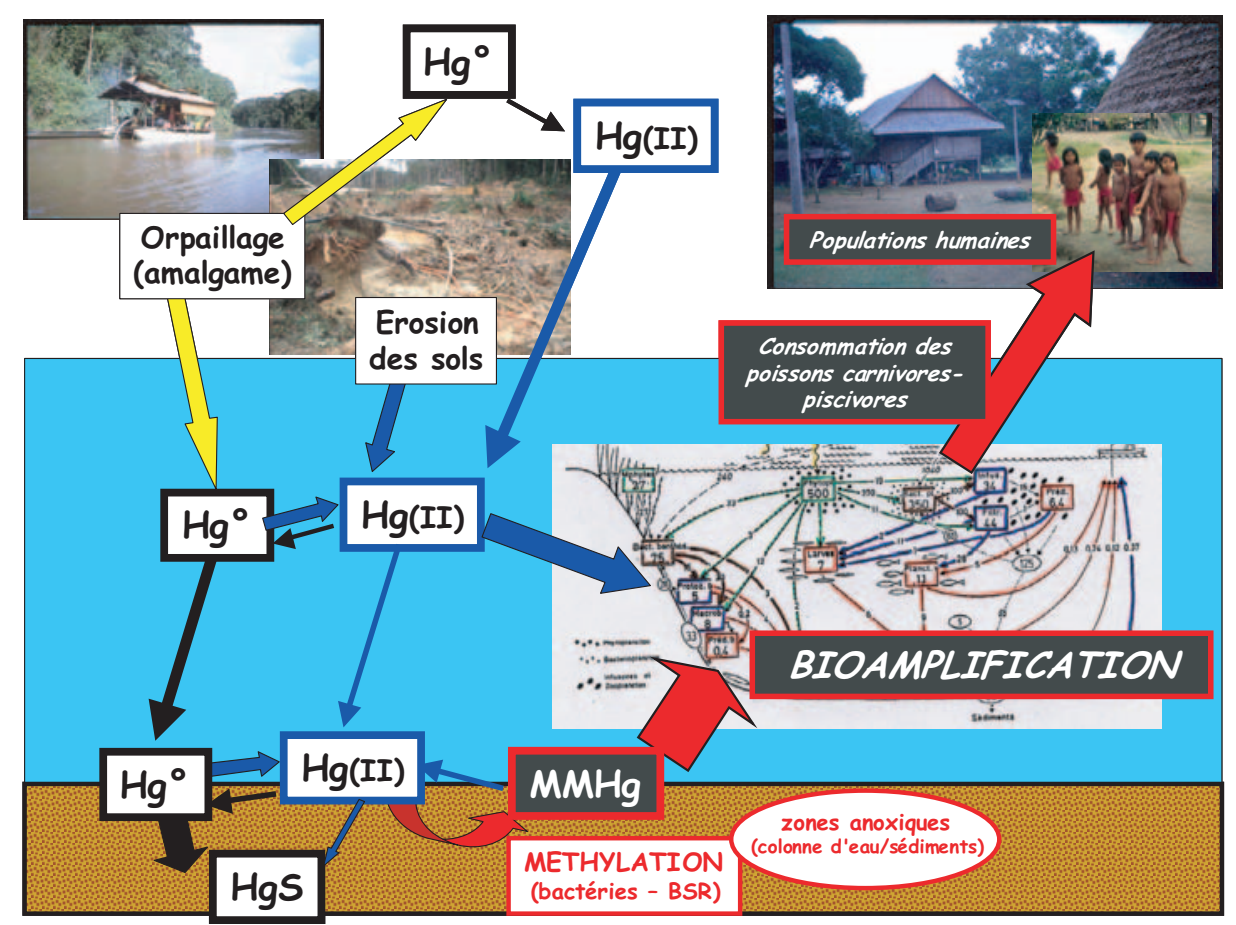

Fig. 2. Principales transformations des formes chimiques du mercure à partir des rejets liés aux activités d'orpaillage et rôle-clef du méthylmercure $(\mathrm{MeHg})$ à l'égard de la contamination des organismes aquatiques et, indirectement, des populations humaines.

BSR : bactéries sulfato-réductrices à l'origine de la méthylation du $\mathrm{Hg}(\mathrm{II})$, en conditions anoxiques $\mathrm{HgS}$ : sulfure de mercure (insoluble).

Fig. 2. Main transformations of the mercury chemical forms with respects to goldmining activities and the key role of the Methylmercury $(\mathrm{MeHg})$ in the contamination of aquatic organisms and human population.

BSR: sulphate-reducing bacteria - HgS: mercury sulfite.

de Saint-Elie à l'amont (Fig. 4 ; ou voir partie 3).

Au sein de la colonne d'eau, les concentrations du Hgtotal(2) sont très faibles : $30 \mathrm{ng} / \mathrm{L}$ (ppt) dans les échantillons non filtrés et $5 \mathrm{ng} / \mathrm{L}$ pour la fraction dissoute $(<0,7 \mu \mathrm{m})$.

\footnotetext{
(2) Hgtotal : ensemble des formes chimiques du mercure présentes dans un échantillon $\left(\mathrm{Hg}^{\circ}\right.$, $\mathrm{Hg}(\mathrm{II}), \mathrm{MeHg}, \ldots)$.
}

Dans les compartiments biologiques, les concentrations dépendent étroitement des niveaux trophiques des espèces échantillonnées : les valeurs moyennes maximales correspondant aux poissons piscivores varient de 4000 à $10000 \mu \mathrm{g} / \mathrm{kg}$ (ppb), sur la base du poids sec (ps) des échantillons. Elles sont environ 50000 fois supérieures à la concentration moyenne globale du métal mesurée dans l'eau 


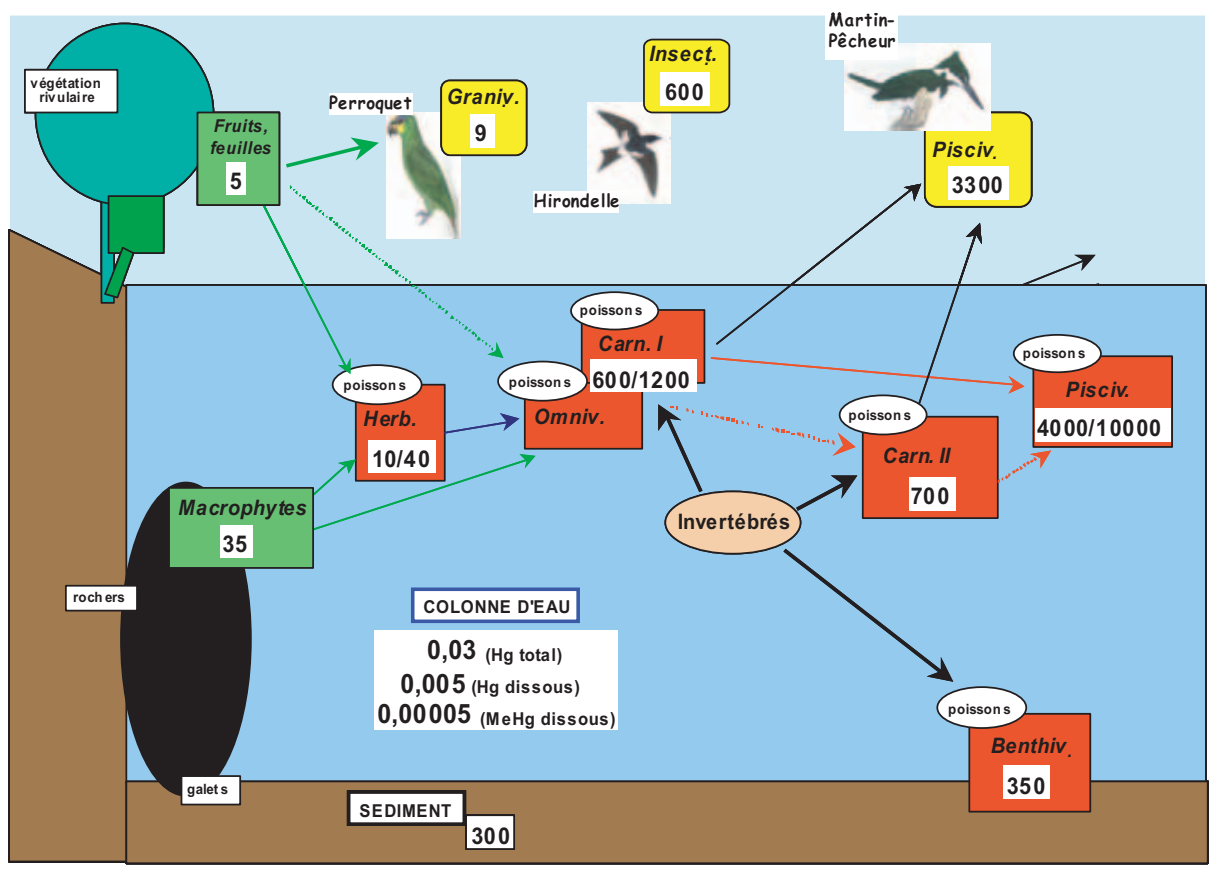

Fig. 3. Représentation schématique des niveaux de contamination par le mercure ([Hgtotal], en $\mu \mathrm{g} / \mathrm{kg}$ ou ppb, poids sec) des principaux niveaux trophiques au sein des communautés de poissons de la rivière Leblond et de trois espèces terrestres. Les concentrations dans la colonne d'eau sont exprimées en $\mu \mathrm{g} / \mathrm{L}$ ou $\mathrm{ppb}$.

Herb : herbivores - Omniv : omnivores - Carn : carnivores - Pisciv : piscivores - Benthiv : benthivores - Graniv : granivores - Insectiv : insectivores.

$\bigcirc$ invertébrés $\square$ poissons.

Fig. 3. Representation of mercury contamination levels ([Hgtotal], $\mu \mathrm{g} / \mathrm{kg}$, dry weight) of the main trophic position within the foodwebs in fish and 3 terrestrial species collected in the Leblond river. Concentrations in the water column are expressed in $\mu \mathrm{g} / \mathrm{L}$.

Herb: herbivorous - Omn: omnivorous - Carn: carnivorous - Pisc: piscivorous - Benth: benthivorous - Graniv: granivorous - Insectiv: insectivorous.

$\bigcirc$ invertebrate $\square$ fish.

et environ 300000 fois plus élevées que la concentration du Hgtotal dans la fraction dissoute, ces facteurs de bioconcentration (FBC) étant calculés sur la base des poids frais (pf) des organismes (pf/ps = 5). Rappelons que la norme de consommation actuellement appliquée sur les continents Nord- et Sud-américains est de $500 \mu \mathrm{g} / \mathrm{kg}$ (pf) ou $2500 \mu \mathrm{g} / \mathrm{kg}$ (ps). À l'opposé, les espèces situées à la base des réseaux trophiques - plantes aquatiques (macrophytes/podostémacées), végétaux rivulaires, espèces herbivores (invertébrés, poissons) - sont caractérisées par des concentrations beaucoup plus faibles (5 à $90 \mu \mathrm{g} / \mathrm{kg}, \mathrm{ps}$ ), soit en moyenne plusieurs centaines de fois 


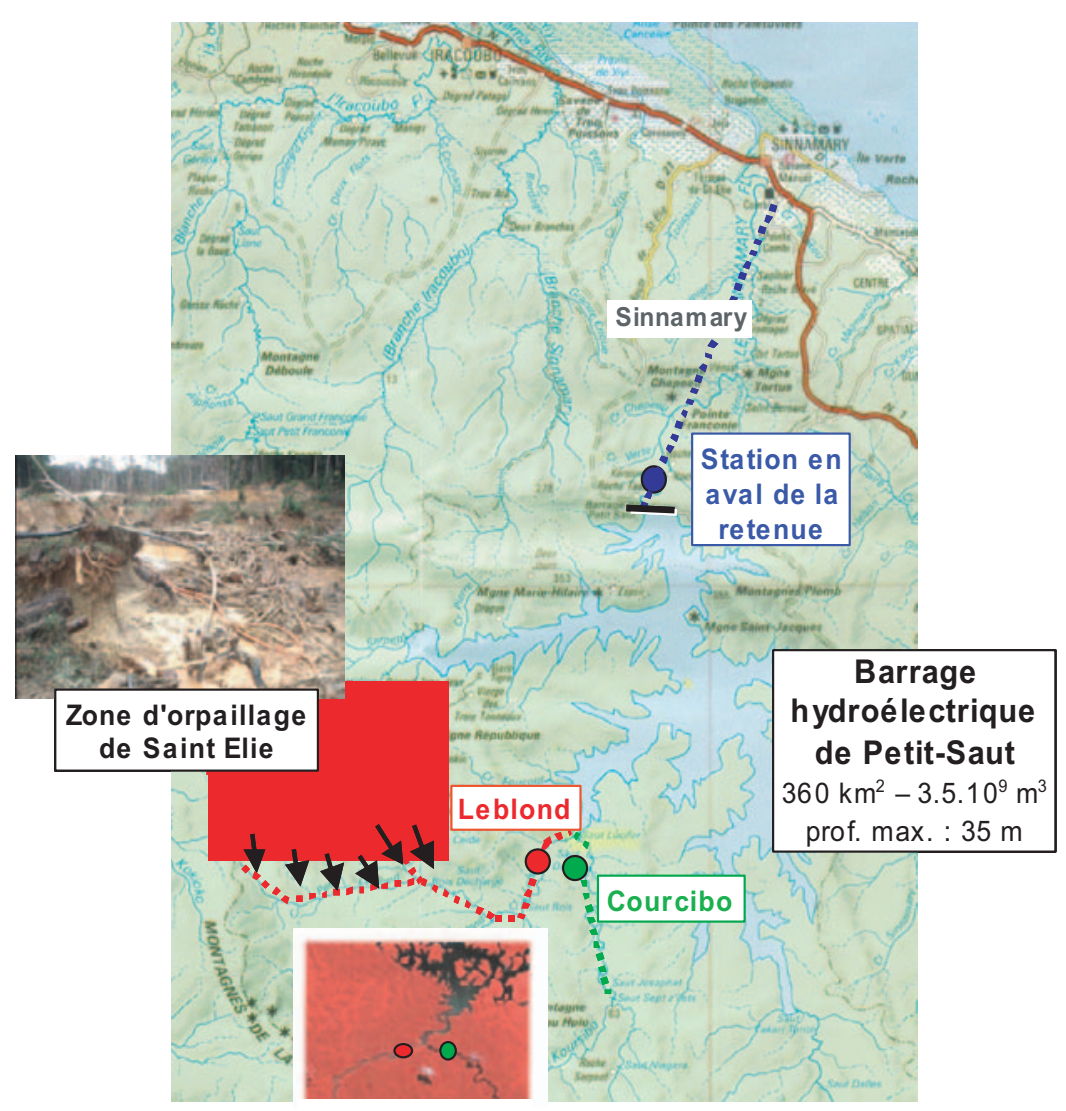

Fig. 4. Localisation des trois stations au niveau de la zone du barrage de Petit-Saut - Leblond, Courcibo et Aval de la retenue - pour analyser les effets de l'orpaillage sur la contamination des réseaux trophiques aquatiques par le mercure.

Photo satellite : CNES/IRD 1998.

Fig. 4. Map of 3 study sites in the Petit-Saut hydroelectric reservoir area - Leblond, Courcibo and downstream of the dam - to describe the goldmining effects on the contamination of the trophic aquatic networks by mercury (photo: CNES/IRD, 1998).

inférieures à celles mesurées dans le tissu musculaire des poissons piscivores.

Parmi les compartiments biologiques étudiés, deux présentent des niveaux de contamination comparativement plus élevés et revêtent ainsi une importance particu- lière au regard de la bioamplification du mercure le long des chaînes trophiques:

- d'une part, les biofilms, correspondant aux communautés périphytiques qui se développent à la surface des substrats solides immergés (épiphyton et épiliton); ils sont constitués 
d'un mélange de particules minérales et organiques, de bactéries, de micro-algues (diatomées) et de protozoaires, avec des concentrations moyennes de mercure de $350 \mu \mathrm{g} / \mathrm{kg}$ (ps). Ces biofilms peuvent représenter des sites privilégiés pour la méthylation du $\mathrm{Hg}(\mathrm{II})$, en accord avec les résultats récemment obtenus sur les microenvironnements réducteurs au sein de la rhizosphère des plantes aquatiques flottantes, au niveau des marécages et des zones inondables du bassin amazonien (Guimaraes et al., 1998) ;

- d'autre part, un ensemble de poissons de petite taille, dénommés communément « yayas » en Guyane, ayant des régimes alimentaires de type «omnivore/carnivore I » et dont les concentrations en Hgtotal dans le muscle sont comprises entre 600 et $1200 \mu \mathrm{g} / \mathrm{kg}$ (ps). Ces poissons représentent la base alimentaire de nombreuses espèces piscivores situées en fin de chaînes trophiques, notamment les aymaras (Hoplias aimara).

Si l'analyse des données de bioaccumulation est effectuée non plus sur la base du Hgtotal mais sur celle du méthylmercure, les facteurs de bioconcentration (FBC) deviennent extrêmement élevés, à cause de la faible proportion du $\mathrm{MeHg}$ dans la fraction dissoute de la colonne d'eau $(0,05 \mathrm{ng} / \mathrm{L}$ en moyenne ou $1 \% \mathrm{du}$ Hgtotal) et, conjointement, de la prédominance de la forme méthylée du mercure dans le tissu musculaire des poissons carnivores (>90\%). Ainsi, pour Hoplias aimara, le FBC [MeHgmuscle]/[MeHgeau.dis.] est supérieur à 20 millions (Durrieu et al., 2005).

Sur la figure 3 sont également représentées quelques espèces terrestres : les résultats montrent très clairement que seuls les animaux dont le régime alimentaire repose partiellement ou totalement sur la consommation de proies aquatiques, sont caractérisés par des concentrations élevées en mercure dans leur tissu musculaire. Ainsi, par exemple, le martin-pêcheur, qui se nourrit quasi-exclusivement de poissons, possède un niveau moyen de contamination proche de celui des poissons piscivores (3300 $\mu \mathrm{g} / \mathrm{kg}$, ps). À l'opposé, les autres espèces, comme les perroquets (régime granivore) ou encore plusieurs espèces de gibier, sont très peu contaminées $([\mathrm{Hg}]<$ $100 \mu \mathrm{g} / \mathrm{kg}$, ps). Ces résultats sont en accord avec les données publiées dans la littérature, montrant de faibles niveaux de contamination des chaînes alimentaires terrestres (Frery et al., 2001 ; Wiener et al., 2002).

\section{ABSENCE DE LIENS DIRECTS ENTRE LES NIVEAUX DE CONTAMINATION DES COURS D'EAU PAR LES ACTIVITÉS D'ORPAILLAGE ET LES CONCENTRATIONS DU MERCURE DANS LES POISSONS - RÔLE-CLEF DU MÉTHYLMERCURE}

Les différentes stations autour du barrage de Petit-Saut ont fourni un excellent support d'étude pour appréhender les relations entre les rejets de mercure liés aux activités d'orpaillage et les niveaux de contamination des hydrosystèmes par les différentes formes chimiques du métal, à la fois dans les compartiments abiotiques (colonne d'eau et sédiments) et biotiques (réseaux trophiques). 
Deux stations ont permis une étude comparative entre deux cours d'eau similaires au regard de leurs caractéristiques hydrologiques mais nettement différenciés par rapport aux activités d'orpaillage (Fig. 4) : (i) la Crique Leblond qui draine une partie importante du bassin-versant de la zone minière de Saint-Elie; (ii) la crique Courcibo, qui ne reçoit pas de rejets en provenance de sites d'orpaillage ${ }^{(3)}$. Une troisième station, à l'aval de la retenue, a servi de référence par rapport à l'impact du barrage sur le Sinnamary, en relation avec les fortes capacités de méthylation du mercure dans la zone anoxique de la colonne d'eau dans la retenue du barrage.

Les résultats des analyses géochimiques au niveau des criques Leblond et Courcibo, notamment la concentration des matières en suspension dans l'eau (MES), et les dosages du $\mathrm{Hg}$ total dans les fractions dissoute et particulaire (Fig. 5) sont en accord avec les impacts attendus des activités d'orpaillage sur les cours d'eau collecteurs : fort accroissement de la turbidité $(\times 5$ en moyenne) et apports de mercure principalement sous forme particulaire (érosion des sols et amalgamation) ; les rapports entre les concentrations moyennes du mercure pour les

(3) Lors des différentes missions réalisées en 1999/2000, la Crique Courcibo était exempte d'activités d'orpaillage, directement sur le lit de la rivière et sur son bassin-versant. Actuellement, d'importants camps d'orpaillage sont installés sur les rives du barrage de Petit-Saut et sur cette crique. II est important de souligner que toute cette zone du Sinnamary a été fortement orpaillée lors de la première ruée vers l'or en Guyane française, à la fin du siècle dernier et au début du $21^{\mathrm{e}}$ siècle.
2 stations «Leblond/Courcibo 》 sont proches de 10 pour le $\mathrm{Hg}$ total (échantillons d'eau non filtrés) et de 4 pour le $\mathrm{Hg}$ particulaire $(>0,7 \mu \mathrm{m})$.

Deux campagnes d'échantillonnage de poissons, réalisées en périodes de hautes et basses eaux, à l'aide de différentes techniques de pêche (filets, roténone), ont permis de capturer 12 espèces communes aux deux stations, les individus de chaque espèce $(N>5)$ ayant des poids similaires. L'analyse statistique des données de bioaccumulation du mercure dans le tissu musculaire (test non paramétrique de Mann-Whitney) révèle une absence de différence significative entre les niveaux de contamination. Les résultats obtenus sur l'espèce $H$. aimara $(N=56)$ sont particulièrement démonstratifs à cet égard (Fig. 6).

Ces résultats, surprenants comptetenu des différences très marquées entre les concentrations de mercure mesurées dans la colonne d'eau des deux rivières, peuvent être interprétés à partir de la similarité des niveaux de contamination de la fraction dissoute par le méthylmercure : Leblond : $0,05 \mathrm{ng} / \mathrm{L}, 1 \%$ Hgtotal et Courcibo : $0,03 \mathrm{ng} / \mathrm{L}, 1,7 \%$ Hgtotal. Ceci traduit des capacités de méthylation comparables entre les deux hydrosystèmes et/ou des taux de transferts du MeHg similaires à partir des sites de méthylation vers la fraction dissoute de la colonne d'eau, cette fraction étant considérée comme la plus biodisponible. Les déplacements des poissons entre les deux criques pourraient également expliquer ces résultats. Toutefois, plusieurs espèces parmi les 12 étudiées sont décrites dans la littérature comme " non migrantes ", notamment $H$. aimara qui effectue des déplacements 


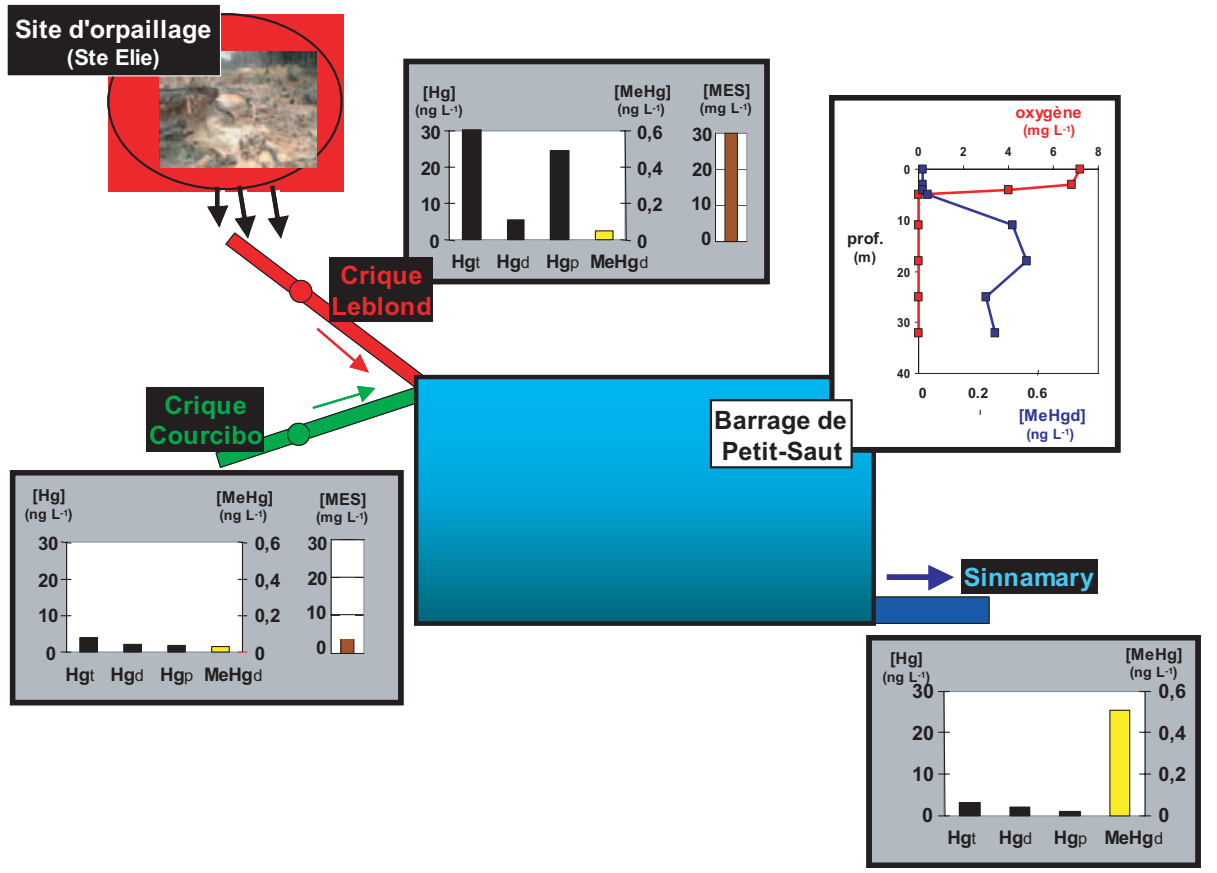

Fig. 5. Représentation synthétique des niveaux de contamination de la colonne d'eau par le mercure et des concentrations en matières en suspension (MES) au niveau des stations de Leblond (activités d'orpaillage), de Courcibo (pas d'activités d'orpaillage) et en aval du barrage de Petit-saut, sur le Sinnamary.

Hgt : ensemble des formes chimiques du mercure dans les échantillons d'eau non filtrés - Hgd : ensemble des formes chimiques du mercure dans les échantillons d'eau filtrés (fraction dissoute $<0,7 \mu \mathrm{m})-\mathrm{Hgp}$ : ensemble des formes chimiques du mercure dans la fraction particulaire $(>0,7 \mu \mathrm{m})$ - MeHgd : concentration du méthylmercure dans la fraction dissoute $(<0,7 \mu \mathrm{m})$.

Fig. 5. Synthetic representation of mercury contamination levels in the water column and suspended particular matter concentrations (SPM) in the rivers Leblond, Courcibo and Petit-Saut downdam zone on the Sinnamary river.

- Hgt: mercury chemical forms in the non filtered water column samples - Hgd: mercury chemical forms in the filtered water column samples (dissolved fraction $<0.7 \mu \mathrm{m}$ )

- Hgp: mercury chemical forms in the particular fraction $(>0.7 \mu \mathrm{m})$ - MeHgd: Methylmercury concentrations in the dissolved fraction $(<0.7 \mu \mathrm{m})$.

transversaux entre le lit des rivières et les zones inondables, lors de la saison des pluies (Goulding, 1980 ; Junk, 1985 ; Roulet \& Maury-Brachet, 2001).

Cette hypothèse est confortée par les résultats obtenus en aval du bar- rage de Petit-Saut (Fig. 4). En effet, les conditions anoxiques de la colonne d'eau de la retenue, au-delà de 4 à $5 \mathrm{~m}$ de profondeur (profondeur maxi : $35 \mathrm{~m}$ ), sont à l'origine d'une importante production de $\mathrm{MeHg}$ 


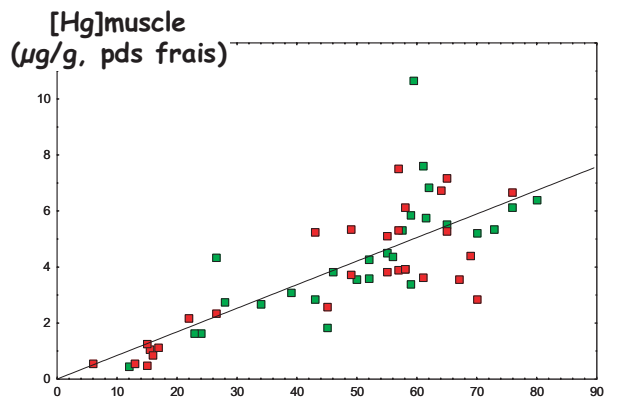

Longueur standard $(\mathrm{cm})$ $\log ([\mathrm{Hg}]$ muscle +1$)$

$(\mu \mathrm{g} / \mathrm{g}, \mathrm{pds}$ frais)

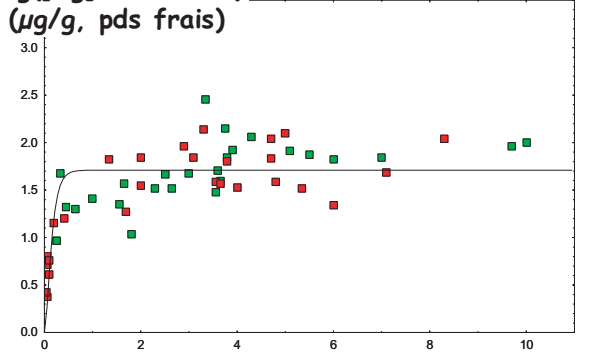

Poids total (kg, pds frais)

Fig. 6. Étude comparée des niveaux de contamination par le mercure du poisson carnivore Hoplias aimara, entre les deux cours d'eau : Leblond (activités d'orpaillage) et Courcibo (pas d'activités d'orpaillage).

Absence de différence significative entre les deux populations de poissons $(N=56)$ : test $U$ de MannWhitney.

Fig. 6. Comparative study of mercury contamination levels of carnivorous fish species Hoplias aimara between the 2 rivers: Leblond (goldmining activities) and Courcibo (no goldmining activities). No significant differences were observed between the two samples $(N=56)$ : Mann-Whitney U-test.

par les bactéries sulfato-réductrices (Rudd, 1995), MeHg qui est ensuite exporté dans le Sinnamary via les rejets d'eau à la sortie des turbines : les concentrations de MeHg juste à l'aval du barrage sont en moyenne de 0,5 ng/L (30 \% du Hgtotal), soit environ dix fois supérieures à celles mesurées dans les criques Leblond et Courcibo (Boudou et al., 2005). L'analyse comparée des concentrations du mercure dans le tissu musculaire de 6 espèces de poissons capturées simultanément au niveau des 3 stations (Fig. 7) met en évidence des différences significatives $(P<0,05)$ et importantes entre la zone aval du barrage et les deux criques situées en amont (orpaillée et non orpaillée), l'écart maximal (facteur 9) étant observé pour l'espèce benthivore Curimata cyprinoïdes, qui se nourrit préférentiellement à partir de débris or- ganiques (Planquette et al., 1996). Ces résultats sont en accord avec les mesures effectuées par le Laboratoire Hydreco le long du Sinnamary, dans le cadre de l'étude d'impact du barrage (Richard et al., 1999, 2000).

\section{CONTAMINATION DES POPULATIONS HUMAINES PAR LE MERCURE : SYNTHĖSE DES DONNÉES \\ SUR LES COMMUNAUTÉS \\ AMÉRINDIENNES DU HAUT-MARONI}

Les études épidémiologiques mises en œuvre par le Réseau National de Santé Publique (RNSP) en 1993/94 sur l'ensemble du territoire guyanais, via le dosage du mercure dans les échantillons de cheveux prélevés au 

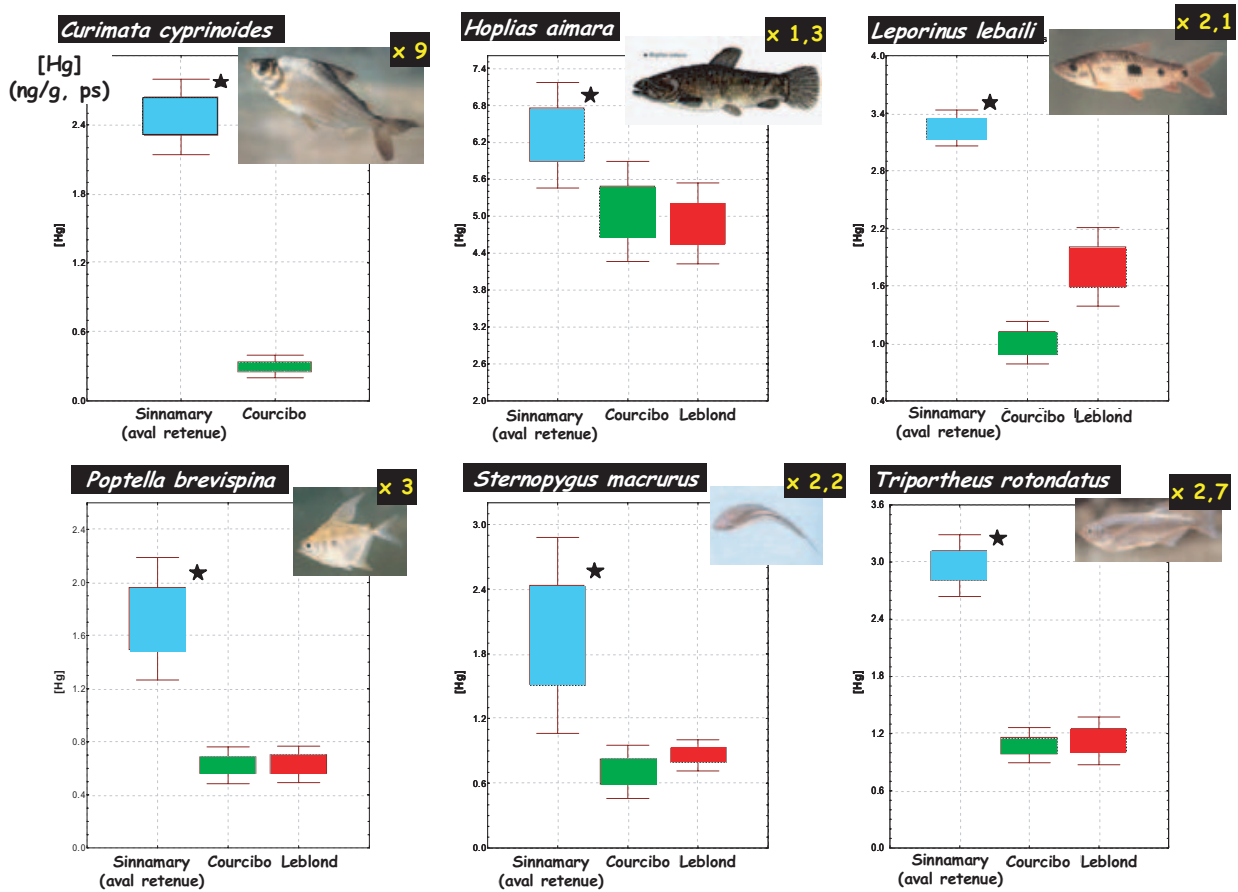

Fig. 7. Étude comparée des niveaux de contamination par le mercure du tissu musculaire de 6 espèces de poissons capturées au niveau des 3 stations : Leblond (activités d'orpaillage), Courcibo (pas d'activités d'orpaillage) et “ Aval du barrage de Petit-Saut » (voir carte Fig. 4). * différences significatives $(P<0,05)$.

Fig. 7. Comparative studies of the mercury contamination levels in the muscle tissue of 6 fish species collected in 3 stations: Leblond (goldmining activities), Courcibo (no goldmining activities) and downstream the Petit-Saut reservoir (see map Fig. 4).

*significant differences $(P<0.05)$.

niveau de 8 centres de collecte, avaient révélé des niveaux de contamination au delà de la norme définie par l'Organisation Mondiale de la santé (OMS - $10 \mu \mathrm{g} / \mathrm{g}$ ) chez les amérindiens vivant dans la zone du HautMaroni (Fig. 1). Les travaux réalisés en 1997 par l'Institut de Veille Sanitaire (InVS), I'INSERM et le LEESA (Université Bordeaux 1/CNRS), au niveau de trois villages amérindiens
(Twenke, Cayode et Antecume-Pata), ont permis (i) de quantifier les apports de mercure liés à la consommation des produits de la pêche et du gibier; (ii) de déterminer sur 235 personnes les niveaux d'imprégnation des populations par le dosage du mercure total et du $\mathrm{MeHg}$ dans les cheveux; (iii) d'effectuer une étude neuropsychologique sur les enfants. Les enquêtes nutritionnelles ont révélé que 
la consommation de poissons chez les Wayanas est bi-quotidienne et l'exposition commence in utero, compte tenu des fortes capacités de transfert du $\mathrm{MeHg}$ au travers de la barrière placentaire. Cette consommation est en moyenne supérieure à $300 \mathrm{~g}$ (pf) de poissons par individu et par jour. Par rapport aux normes préconisées par plusieurs groupes d'experts (OMS, FAO) qui recommandent un apport hebdomadaire de mercure inférieur à $300 \mu \mathrm{g}$ pour le Hgtotal et $200 \mu \mathrm{g}$ pour le $\mathrm{MeHg}$, la quasi-totalité des habitants des villages amérindiens âgés de plus de 7 ans dépasse ces valeurs limites. Les analyses de mercure effectuées sur 242 échantillons de poissons consommés par ces populations ont révélé que $15 \%$ des échantillons étaient supérieurs à la norme de $0,5 \mu \mathrm{g} \cdot \mathrm{g}^{-1}$ (pf). Seulement 4 espèces de poissons carnivores, correspondant à $28 \%$ des quantités de poissons consommées, contribuaient à plus de $70 \%$ des teneurs de mercure ingérées. Les analyses effectuées sur les cheveux ont clairement démontré la corrélation entre les niveaux d'imprégnation des populations par le $\mathrm{MeHg}$ et le régime alimentaire : les personnes à risque sont de fortes consommatrices de poissons carnivores (Frery et al., 2001). Toutefois, la comparaison de l'ensemble des espèces de poissons capturées sur les différents sites de Guyane, sur la base de critères biométriques similaires (poids/longueur), montre que les concentrations moyennes du mercure dans les poissons du Haut-Maroni sont plus faibles que celles mesurées sur les différentes stations au niveau du barrage de Petit-Saut et en aval de la retenue. De même, pour les végétaux et les invertébrés aquatiques, les résultats de bioaccumulation ne mettent pas en évidence de différences globalement significatives par rapport aux autres sites d'étude, les concentrations sur certains taxons étant nettement inférieures pour Antecume Pata (invertébrés brouteurs et filtreurs par exemple). Ces données relatives aux compartiments biologiques sont en accord avec les analyses géochimiques effectuées en 2001, qui traduisent des teneurs en mercure relativement homogènes entre les différentes stations en amont et en aval des villages amérindiens et comparativement plus faibles que celles mesurées sur la zone de Petit-Saut. II est évident que les niveaux d'imprégnation des populations amérindiennes, estimés via les dosages du mercure dans les cheveux, résultent des effets combinés des niveaux de contamination des produits de la pêche, plus précisément des espèces situées au sommet des réseaux trophiques aquatiques, et de l'abondance et de la fréquence des repas à base de poissons.

Parallèlement aux enquêtes nutritionnelles et aux estimations des teneurs de mercure apportées journalièrement ou hebdomadairement par la consommation des produits de la pêche, des études neurologiques et neuropsychologiques ont été menées à l'initiative de I'INSERM et de l'InVS sur les populations d'enfants Wayanas (Cordier \& Garel, 1999). Les conséquences neurotoxiques sur les enfants ne traduisent pas pour le moment de déficiences majeures, en comparaison par exemple avec les signes cliniques observés lors de l'intoxication de Minamata, au Japon (Clarkson, 1992; Hanada et al., 1997). Toutefois, 
l'analyse des atteintes précoces du système nerveux, grâce à l'utilisation de batteries de tests traduisant les perturbations des fonctions motrices, sensorielles, émotionnelles ou cognitives, ou encore les déficits de l'organisation visuo-spatiale, révèlent des effets significatifs, directement corrélés aux niveaux d'imprégnation des individus par le mercure (Cordier \& Garel, 1999). Les déficits observés sont similaires à ceux décrits au Brésil, qui montrent des dysfonctionnements au niveau de la coordination et de la rapidité des mouvements fins, avec une diminution de sensibilité aux contrastes visuels (Dolbec et al., 2000 ; Grandjean et al., 1999; Lebel et al., 1996). Des effets cytogénétiques, à partir de la détermination de l'index mitotique sur des globules blancs, ont été récemment mis en évidence sur des populations brésiliennes des rives du Tapajos, les effets étant une nouvelle fois fortement corrélés aux teneurs en $\mathrm{Hg}$ dans les cheveux (Amorim et al., 2000).

Dans ce contexte, une consommation « raisonnée » des poissons, éliminant ou réduisant les espèces carnivores/piscivores, représente une mesure efficace pour limiter très significativement les apports trophiques de $\mathrm{MeHg}$ et, conjointement, les niveaux d'imprégnation des populations et les risques neurotoxiques qui peuvent en résulter. Soulignons qu'une telle mesure doit être appliquée en priorité aux femmes enceintes, eu égard au transfert de la forme organique du métal vers le fœtus, via la traversée de la barrière placentaire. II faut toutefois insister sur le fait que les aspects socio-culturels concernant les activités de pêche et la consommation des poissons par les amérindiens séden- tarisés dans la zone du Haut-Maroni doivent être pris en compte. Seules des campagnes d'information pertinentes et adaptées, mises en œuvre en étroite relation avec les habitants des différents villages, et parallèlement des études démontrant le bien-fondé de ces mesures (diminution significative des concentrations du $\mathrm{Hg}$ dans les cheveux, par des campagnes de suivi), peuvent conduire à des résultats efficaces à tous égards.

Notons in fine qu'une politique cohérente et efficace d'organisation et de contrôle des activités d'orpaillage en Guyane s'avère de plus en plus nécessaire, plusieurs solutions techniques étant disponibles pour limiter, voire supprimer dans certains cas, les rejets de mercure dans l'environnement. Comme indiqué par C. TaubiraDelannon dans son rapport «L'or en Guyane : éclats et artifices » (2001), il est absolument nécessaire de traiter simultanément l'ensemble des problèmes économiques, sociaux, culturels, environnementaux et politiques posés par l'orpaillage.

\section{REMERCIEMENTS}

Ces recherches ont été financées par le Programme «Environnement, Vie et Sociétés » du CNRS, le Ministère de l'Environnement et les fonds européens FEDER (Préfecture de Guyane). Les missions sur le terrain ont bénéficié de la collaboration de la Compagnie "Chasse et Pêche », du Laboratoire Hydreco, de l'Équipe « Hydrobiologie " de l'IRD (Cayenne), de la Mission pour la création du Parc de Guyane et des habitants des villages Wayanas sur le Haut Maroni. Les analyses de Hgtotal et de $\mathrm{MeHg}$ dans les échantillons d'eau ont été réalisées par S. Azemard (AIEA, Monaco). 


\section{RÉFÉRENCES BIBLIOGRAPHIQUES}

Amorim M.I.I., Mergler D., Bahia M.O., Dubeau H., Miranda D., Lebel J., Burbano R.R. \& Lucotte M. 2000. Cytogenetic damage related to low levels of methylmercury contamination in the Brazilian Amazon. An. Acad. Bras. 72: 497-507.

Bidone E.D., Castilhos Z.C., Santos T.L.L., Sousa T.M.M. \& Lacerda L.D.D. 1997. Fish contamination and human exposure to mercury in Tartarugalzinho river, AMAPA State, Northern Amazon, Brazil. A screening approach. Wat. Air Soil Pollut. 97: 9-15.

Boischio A.A.A. \& Henshel D.S. 1996. Risk assessment of mercury exposure through fish consumption by the riverside people in the Madeira Basin, Amazon, 1991. Neuro Toxicol. 17: 169176.

Boudou A. \& Ribeyre F. 1997. Mercury in the food web. Accumulation and transfer mechanisms. Mercury and its effects on Environment and Biology, "Metal ions in biological systems" series, Vol. 34. A. Sigel and $H$. Sigel eds.: pp. 289-319. Marcel Dekker (New York).

Boudou A., Maury-Brachet R., Coquery M., Durrieu G. \& Cossa D. 2005. Synergic effect of gold mining and damming on mercury contamination in fish. Environ. Sci Technol. 39: 2448-2454.

Carmouze J.P., Lucotte M. \& Boudou A. - Le mercure en Amazonie: rôle de l'homme et de l'environnement risques sanitaires. Série "Expertises collégiales", IRD Editions (Paris), 494 p.

Clarkson T.W. 1992. Mercury: major issues in environmental health. Env. Health Persp. 100: 31-38.

Cordier S. \& Garel M. 1999. Risques neurotoxiques chez l'enfant liés à l'exposition au méthylmercure en Guyane Française. IVS (Paris), 52 p.

Dolbec J., Mergler D., Sousa Passos C.J., Sousa de Morais S. \& Lebel J. 2000.
Methylmercury exposure affects motor performance of a riverine population of the Tapajos river, Brazilian Amazon. Interna. Arch. Occup. Environ. Health 73: 195-203.

Dorea J.G., Moreira M.B., East G. \& Barbosa A.C. 1998. Selenium and mercury concentrations in some of fish species of the Madeira river, Amazon Basin, Brazil. Biol. Trace Elem. Res. 65: 211-220.

Durrieu G., Maury-Brachet R. \& Boudou A. 2005. Goldmining and mercury contamination of the piscivorous fish Hoplias aimara in French Guiana (Amazon Basin). Ecotox. Environ. Safety 60: 315-323.

Fréry N., Maillot E., Deheeger $M$., Boudou A. \& Maury-Brachet R. 1999. Exposition au mercure de la population amérindienne Wayana de Guyane Enquète alimentaire. IVS (Paris), 82 p.

Fréry N., Maury-Brachet R., Maillot E. Deheeger M., de Mérona B. \& Boudou A. 2001. Goldmining activities and mercury contamination of native Amerindian communities in French Guiana: key role of fish in dietary uptake. Environ. Health Persp. 109: 449-456.

Goulding M. 1980. The fishes and the forest: explorations in Amazonian natural history. Univ. California Press, Berkeley (USA).

Grandjean P., White RF., Nielsen A., Cleary D. \& de Oliveria Santos E.C. 1999. Methylmercury neurotoxicity in Amazonian children downstream from gold mining. Environ. Health Persp. 107: 587-591.

Grimaldi M. \& Grimaldi C. 2002. The role of pedogenesis processes in the spatial variability of yotal mercury in French Guiana soils. Geoderma (in press).

Guimaraes J.R.R., Meili M., Malm O. \& de Sousa Brito E.M. 1998. Hg methylation in sediments and floating meadows of a tropical lake in the Pantanal floodplain, Brazil. Scien. Tot. Environ. 213: 165175. 
Hanada R., Arimura K. \& Osame M. 1997. Maternal-fetal mercury transport and fetal mercury poisoning. Mercury and its effects on Environment and Biology, "Metal ions in biological systems" series, Vol. 34. A. Sigel and H. Sigel eds.: 405-420. Marcel Dekker (New York).

Junk W.J. 1985. Tempory fat storage, an adaptation of some fish species to the water level fluctuations and related environmental changes of the Amazon river. Amazoniana 9: 315-351.

Lacerda de L.D. \& Salomons W. 1998. Mercury from gold and silver mining: a chemical time bomb ? Springer Verlag (Berlin), 146 p.

Lebel J., Mergler D., Lucotte M., Amorin M., Dolbec J., Miranda D., Arantes G., Rhéault I. \& Pichet P. 1996. Evidence of early nervous system disfunction in Amazonian populations exposed to low levels of methylmercury. Neurotoxicol. 17: $157-168$.

Malm O. 1998. Gold mining as a source of mercury exposure in the Brazilian Amazone. Environ. Res. 77: 73-78.

Mason R., Reinfelder J.R. \& Morel F.M.M. 1995. Bioaccumulation of mercury and methylmercury. Wat. Air Soil Pollut. 80: 915-921.

Maury-Brachet R., Durrieu G., Dominique Y. \& Boudou A. 2006. Mercury distribution in fish organes and food regimes: significant relationships from twelve species collected in French Guiana (Amazon basin) Sci Total Environ. 368: 262-270.

Moreira J.C. 1996. Threats by heavy metals: human and environmental contamination in Brazil. Sci. Total Environ. 188: 61-71.

Morel F.M.M., Kraepiel A.M.M. \& Amyot M. 1998. The chemical cycle and bioaccumulation of mercury. Ann. Rev. Ecol. Syst. 29: 543-566.

Picot J.C., Foucher J.L. \& Wagner R. 1993. Production aurifère et mercure utilisé, de l'origine à nos jours. BRGMGuyane, Rapport R37837, 18 p. + annexes.
Planquette P., Keith P. \& Le Bail P.Y. 1996.- Atlas des poissons d'eau douce de Guyane (tome 1). Collection du Patrimoine naturel, Vol. 22 , MNHN/INRA/Min. Envt, Paris, 429 p.

Reuther R. 1994. Mercury accumulation in sediment and fish from rivers affected by alluvial gold mining in the brazilian Madeira river basin, Amazon. Environ. Monitor. Assess. 32: 239-258.

Richard S., Arnoux A., Cerdan P. \& Horeau V. 1999. Mercure et poissons: influence de la création d'un réservoir hydroélectrique en milieu néotropical (fleuve Sinnamary, Guyane Française). Congrès AFL/UOF, Bordeaux.

Richard S., Arnoux A., Cerdan P., Reynouard C. \& Horeau V. 2000. Mercury levels of soils, sediments and fish in French Guiana, South America. Water, Air and Soil Pollut. 124: 221-244.

Roulet M. \& Lucotte M. 1995. Geochemistry of mercury in pristine and flooded ferralitic soils of a tropical rain forest in French Guiana, South America. Water, Air and Soil Pollut. 80: 1079-1088.

Roulet M. \& Maury-Brachet R. 2001. Le mercure dans les organismes aquatiques amazoniens. Le Mercure en Amazonie. Carmouze J.P., Lucotte, M. et Boudou, A. eds.: 203-271 (IRD, Paris).

Roulet M., Lucotte M., Canuel R., Farella N., de Freitos Gog Y.G., Peleja J.R., Guimaraes J.R.R., Mergler D. \& Amorim M. 2001. Spatio-temporal geochemistry of $\mathrm{Hg}$ in waters of the Tapajós and Amazon rivers, Brazil. Limnol. Oceanogr. 46: 1158-1170.

Roulet M., Lucotte M., Guimaraes J.R.R. \& Rheault I. 2000. Methylmercury in water, seston and epiphyton of an Amazonian river and its floodplain, Tapajós River, Brazil. Sci. Total Environ. 261: 43-59.

Roulet M., Lucotte M., Saint Aubin A., Tran S., Rhéault I., Farella N., Jesus Da Silva E., Dezaencourt J., Sousa Passos 
C.-J., Santos Soares G., Guimaraes J.R.D., Mergler D. \& Amorin M. 1998. The geochemistry of mercury in central Amazonian soils developed on the Alter-do-Chao formation of the lower Tapajos River Valley, Para state, Brasil. Sci. Total Environ. 223: 1-24.

Rudd J.W.W. 1995. Sources of methylmercury to freshwater ecosystems: a review. Wat. Air Soil Pollut. 80: 697-713.

Veiga M.M., Hinton J. \& Lilly C. 1999. Mercury in the Amazon: a comprehensive review with special emphasis on bioaccumulation and bioindicators. Proc. NIMD Forum'99: 19-39.

Villas Bôas R.C. 1997. The mercury problem in the Amazon due to gold extraction. J. Geoch. Explo. 58: 217-222.

Wiener J.G., Krabbenhoft D.P., Heinz G.H. \& Scheuhammer A.M. 2002. Ecotoxicology of mercury. Handbook of Ecotoxicology. D.J. Hoffman, B.A. Rattner, G.A. Burton and J. Cairns eds. (CRC Press, Boca Raton, USA). 